\title{
Isolated aberrant right cysticohepatic duct injury during laparoscopic cholecystectomy: Evaluation and treatment challenges of a severe postoperative complication associated with an extremely rare anatomical variant
}

\author{
Konstantinos Vasiliadis ${ }^{1}$, Elena Moschou ${ }^{1}$, Sofia Papaioannou ${ }^{2}$, Panagiotis Tzitzis ${ }^{1}$, Albion Totsi ${ }^{1}$, \\ Stamatia Dimou', Eleni Lazaridou ${ }^{3}$, Dimitrios Kapetanos ${ }^{4}$, and Christos Papavasiliou ${ }^{1}$ \\ ${ }^{1}$ First Surgical Department and ${ }^{2}$ Department of Radiology, General Hospital Papageorgiou, \\ ${ }^{3}$ School of Medicine, Aristotle University of Thessaloniki, ${ }^{4}$ Department of Gastroenterology, \\ General Hospital Papanikolaou, Thessaloniki, Greece
}

\begin{abstract}
A typical bile duct branching patterns represent one of the major causes of bile duct injury (BDI) during laparoscopic cholecystectomy (LC). The most common classified variations of bile duct branching, involve the right posterior sectoral duct (RPSD) and its joining with the right anterior or left hepatic duct. Variant bile duct anatomy can rarely be extremely complex and unclassified. This report describes an extremely rare case of an isolated injury to an aberrant right hepatic duct formed by the joining of ducts from segments V, VII, and VIII draining into the cystic duct (cysticohepatic duct) during LC, associated with an inferior RPSD opening to left hepatic duct. Detailed evaluation of both endoscopic and magnetic cholangiograms established the diagnosis. Bile duct injury was subsequently managed surgically by a demanding Roux-en-Y hepaticojejunostomy. This extremely rare case aims to serve as a useful reminder of the consistent inconsistency of biliary anatomy, alerting surgeons to beware of variant bile duct branching patterns during open or LC that constitute a dreadful pitfall for severe and life-threatening bile duct injuries. (Ann Hepatobiliary Pancreat Surg 2020;24:221-227)
\end{abstract}

Key Words: Laparoscopic cholecystectomy; Bile duct injury; Right sectoral bile duct; Hepaticojejunostomy; Variability in bile duct branching pattern; Rule rather than the exception

\section{INTRODUCTION}

Iatrogenic bile duct injuries (BDI) still remain one of the most devastating complications during open or laparoscopic cholecystectomy (LC). ${ }^{1}$ They usually occur as a consequence of misidentification of anatomical structures in the cystohepatic triangle and the presence of atypical-anomalous biliary tree branching patterns. ${ }^{2}$ The most common classified variations of bile duct branching involve the right posterior sectoral duct (RPSD) and its joining with the right anterior sectoral (RASD) or left hepatic duct. ${ }^{3}$ Among bile duct branching pattern variations, anomalous opening of an aberrant right hepatic duct into the cystic duct (cysticohepatic duct) represents a rare and extremely dangerous bile duct configuration, given its high vulnerability to injury during cholecystectomy. Very rare, bile duct branching pattern anomalies are complex, making a routine procedure such as LC, a true surgical challenge, if devastating complications such as inadvertent ligation or resection of major bile ducts are to be avoided.

This report describes the challenging diagnostic and therapeutic management of an extremely rare case of an isolated injury to an aberrant right hepatic duct formed by the joining of ducts from segments V, VII, and VIII draining into the cystic duct (cysticohepatic duct) during LC, associated with an inferior RPSD opening to left hepatic duct.

Received: September 13, 2019; Revised: January 15, 2020; Accepted: January 20, 2020

Corresponding author: Konstantinos Vasiliadis

First Department of General Surgery, Papageorgiou Hospital, GR-56 403, West Ring Road, Nea Efkarpia, Thessaloniki, Greece Tel: +30-2313-323556, Fax: +30-2313323587, E-mail: konvasisurg@gmail.com

Copyright (C) 2020 by The Korean Association of Hepato-Biliary-Pancreatic Surgery

This is an Open Access article distributed under the terms of the Creative Commons Attribution Non-Commercial License (http://creativecommons.org/ censes/by-nc/4.0) which permits unrestricted non-commercial use, distribution, and reproduction in any medium, provided the original work is properly cited. Annals of Hepato-Biliary-Pancreatic Surgery • pISSN: 2508-5778 - eISSN: 2508-5859 


\section{CASE}

A 46-year-old female patient underwent LC following an episode of acute cholecystitis at an outside hospital. The patient had an uneventful postoperative course and was discharged on the second postoperative day (POD). Index operation was deemed to be unremarkable. On the 7th POD the patient presented to the emergency department at the same institution, with severe pain in the right hypochondrium which, radiated to her back, associated with abdominal distention. Physical examination revealed mild abdominal tenderness and fever $\left(38.8^{\circ} \mathrm{C}\right)$. Blood analyses revealed mild elevation of total bilirubin $(2.4$ $\mathrm{mg} / \mathrm{dl})$ and serum C-reactive protein $(1 \mathrm{mg} / \mathrm{dl})$, while the other laboratory values including liver and pancreas function tests were normal. An abdominal ultrasound revealed fluid collection in the hepatorenal recess. Contrast enhanced computed tomography (CT) scan was performed depicting free fluid in the Morison's pouch, perihepatic and right subphrenic spaces. No free peritoneal air, biliary duct dilatation or radiopaque residual biliary calculi were seen. Based on clinical presentation, physical examination, and imaging findings a presumed diagnosis of a post-cholecystectomy bile leakage was made.

Following CT, clinical reevaluation revealed abdominal rigidity necessitating an urgent laparoscopy which revealed free peritoneal bile collection. Conversion to a formal laparotomy through a right Kocher incision was decided, to meticulously explore the site of bile leakage. A slow continuous ooze of bile was noted in the infundibulum bed, which was interpreted as a subsegmental minor accessory bile duct leak and managed by clip ligation. No signs of bile leakage from the principal extrahepatic bile ducts was noted. A surgical drain was placed into the gallbladder fossa-Morison's pouch.

The following days no bilious fluid output from the surgical drain was evident. The surgical drain remained in place continuing to drain a minimal amount of serous fluid and was completely removed on the 5th POD. On the 6th POD right upper quadrant pain recurs associated with a Kehr's sign and the presence of a small amount of bile stained fluid extrusion through a small dehiscence in the middle part of the surgical wound. An urgent magnetic resonance imaging (MRI)-magnetic resonance cholangiopancreatography (MRI-MRCP) was performed de- picting an aberrant, non-dilated right sectoral hepatic duct draining into the infundibulum fossa, in addition to bile leakage in that site, left subhepatic and right subphrenic spaces. The common hepatic duct, bile duct, and cystic duct stump appeared normal. Based on these findings a major BDI was evident and the patient was transferred to our department.

Upon arrival to our clinic, the patient was hemodynamically stable, afebrile, with no acute distress. Physical examination revealed mild tenderness in the right hypochondrium and periumbilical region, a posistive Kehr's sign and the presence of small amount of bile extruding through the surgical wound. Initial laboratory test results were: alanine aminotransferase: $35 \mathrm{U} / \mathrm{L}$ (normal: 7-40 $\mathrm{U} / \mathrm{L})$; aspartate aminotransferase: $40 \mathrm{U} / \mathrm{L}(7-40 \mathrm{U} / \mathrm{L})$; alkaline phosphatase: $360 \mathrm{U} / \mathrm{L}$ (normal: 39-117 U/L); total bilirubin: $2.3 \mathrm{mg} / \mathrm{dl}$ (normal: $0.2-1.3 \mathrm{mg} / \mathrm{dl}$ ); and direct bilirubin: $0.5 \mathrm{mg} / \mathrm{dl}$ (normal: $0.0-0.3 \mathrm{mg} / \mathrm{dl}$ ).

An urgent repeat MRI-MRCP (Fig. 1) scan performed at our institution suggested an isolated injury to an aberrant right hepatic duct with consequent right anterior section and superior segment of right posterior sector bile leak and biloma formation in the left subhepatic and mainly in the right subphrenic space, combined with a right posterior inferior duct variation anomaly with no concomitant vascular injuries. Additional findings were

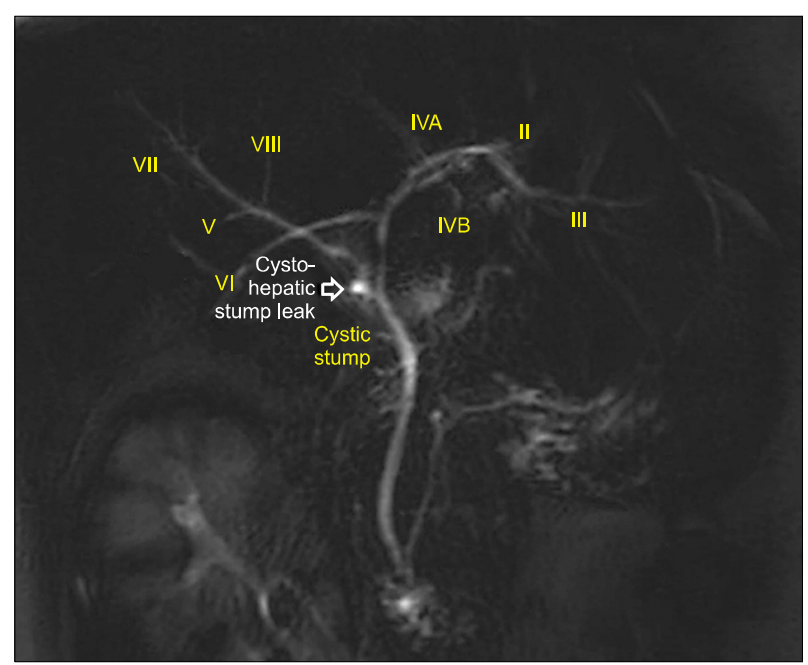

Fig. 1. Magnetic resonance cholangiopancreatography depicting an isolated injury to an aberrant right hepatic duct with consequent right anterior section and superior segment of right posterior sector bile leak, combined with a right posterior inferior duct variation anomaly. 
fluid collections in the gallbladder fossa, in the region of the ligamentum teres and a third fluid collection $10 \mathrm{~cm}$ in diameter, located in the subphrenic space extending into the umbilical fissure of the liver. Subsequently the patient underwent an urgent CT-guided percutaneous drain placement in the right subphrenic space which drained $260 \mathrm{ml}$ of bilious fluid.

The following day the patient underwent an ERCP that revealed normal in size intrahepatic and extrahepatic ducts. No stones, strictures, or leaks were identified during the study, which in general was interpreted as being normal (Fig. 2). Because of high bilious fluid output from the per-

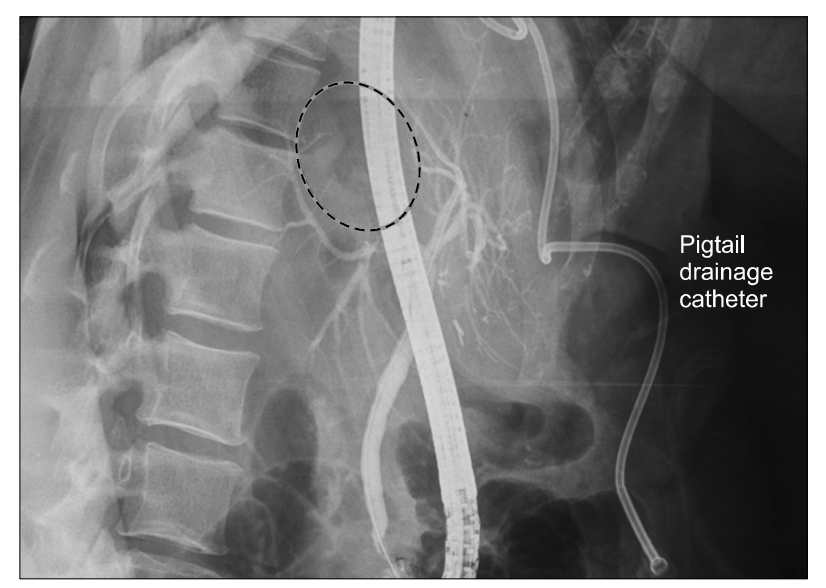

Fig. 2. Endoscopic retrograde cholangiography depicting normal findings apart from paucity of intrahepatic biliary filling, mainly in the right anterior liver section (area surrounded by a dotted line) providing indirect diagnostic signs of a possible sectoral duct injury.

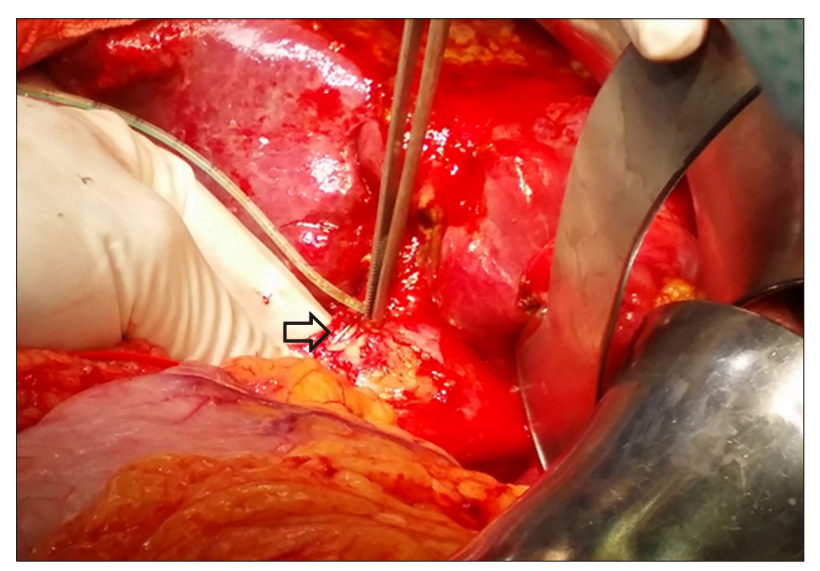

Fig. 3. Intraoperative photograph. Identification and intubation of a bile leaking duct stump, measuring roughly 3 $\mathrm{mm}$ in diameter, located $\sim 0.5 \mathrm{~cm}$ antero-laterally to the clipped cystic duct stump (arrow). cutaneous CT-guided placed pig-tail catheter, a 10-French biliary stent was placed in the right biliary ductal system following endoscopic sphincterotomy (ES). Regardless of ERCP-ES and stent placement, the daily bilious output failed to fall, averaging $180 \mathrm{ml} /$ day. Detailed evaluation of both endoscopic and magnetic cholangiograms suggested the possibility of a disconnected aberrant right hepatic duct as the cause of bile leak. Therefore, on the 9th POD the patient underwent an exploratory laparotomy for the management of a suspected aberrant right bile duct injury.

During the operation a meticulous and challenging dissection was performed at hilar plate and hepatoduodenal ligament. Exploration achieved identification of a bile leaking duct stump, corresponding to the stump of the transected aberrant right hepatic duct seen at MRCP, which was measuring $\sim 3 \mathrm{~mm}$ in diameter, located $\sim 0.5$ $\mathrm{cm}$ antero-laterally to the clipped cystic duct stump (Fig. 3). The cystic stump was the only duct stump identified in the perimeter of hepatic and common bile duct. Based on operative findings it became evident that the aberrant right hepatic duct was running parallel to the common channel of left hepatic and RPSD opening into to the cystic duct through the Calot's triangle (Fig. 4). Intraoperative cholangiograms (IOC) through the supposed aberrant right hepatic ductal stump confirmed that it was indeed an anomalous right hepatic duct that filled segments V, VIII and VII of the right hemi-liver (Fig. 5A). No concomitant BDI were visualized on IOC performed through the com-

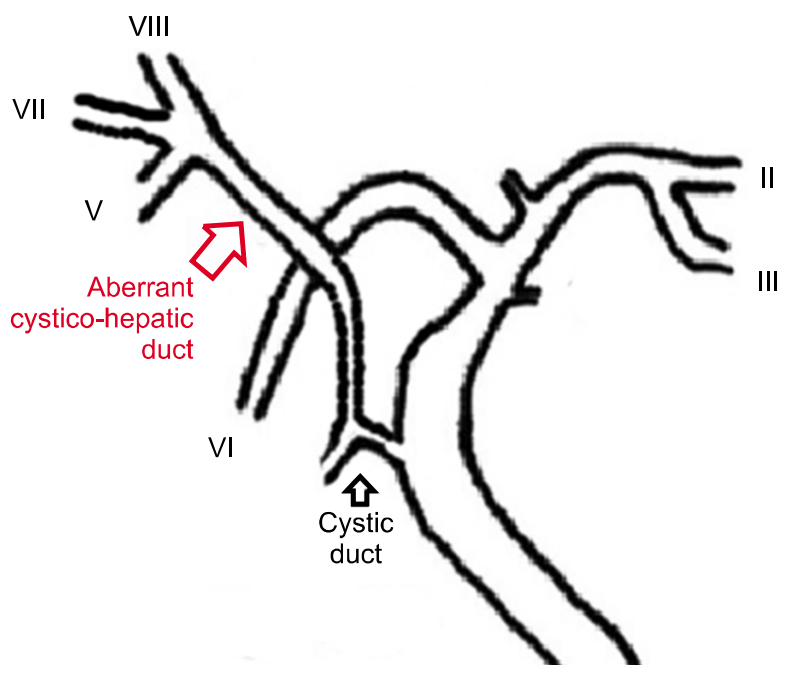

Fig. 4. Schematic drawing of intrahepatic and extrahepatic biliary anatomy of the present case. 

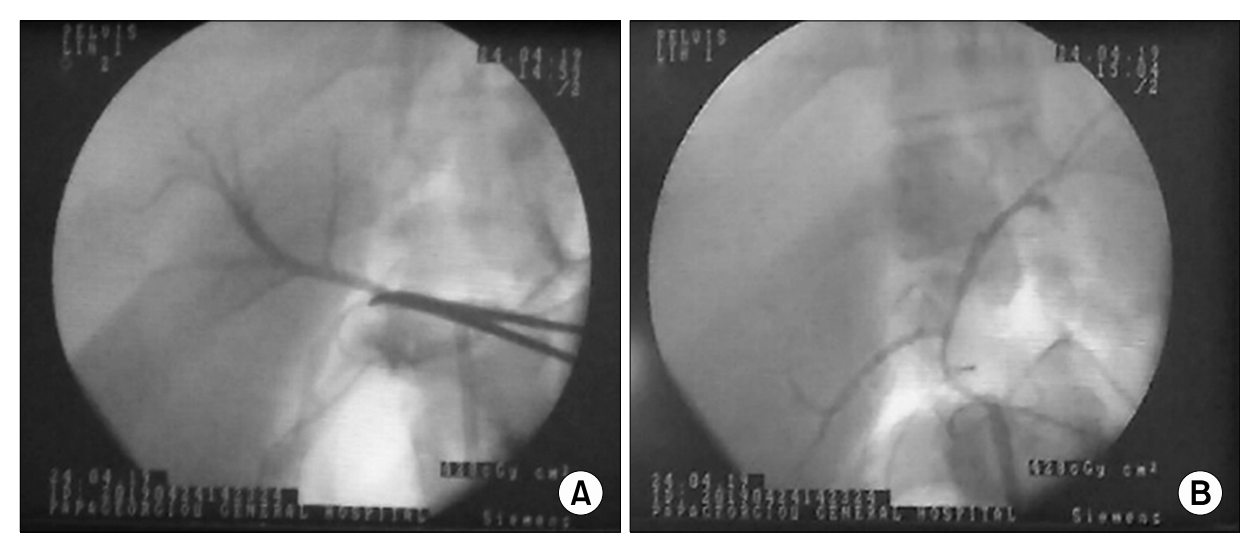

Fig. 5. Intraoperative cholangiogram through the stump of the injured duct (A) confirms complete transection of an anomalous right hepatic duct that filled segments V, VIII and VII of the right hemi-liver, and through the common bile duct (B) depicting no concomitant bile duct injuries.

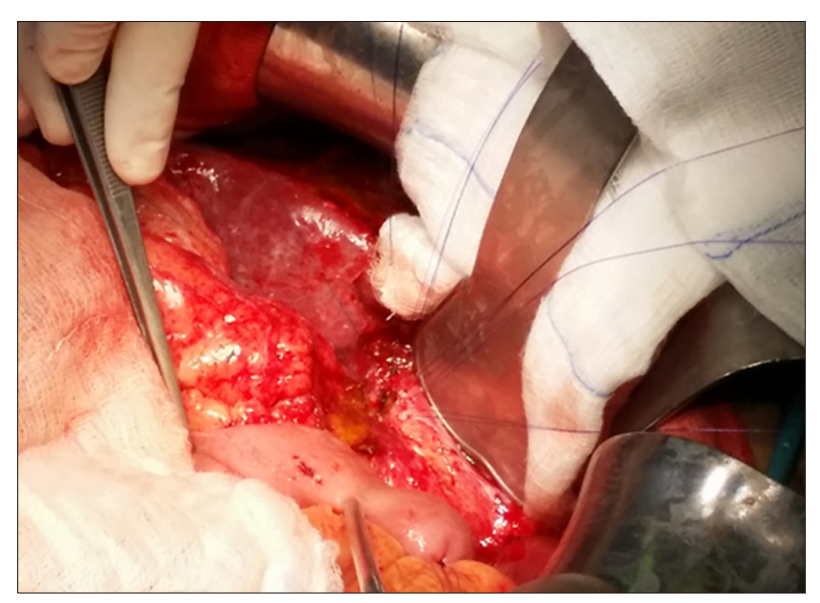

Fig. 6. Intraoperative photograph. Following meticulous dissection and debridement of the aberrant cystico-hepatic duct stump, a $65-\mathrm{cm}$ in length transmesocolic, retrogastric Roux-en-Y limb was anastomosed termino-laterally with the aberrant biliary duct stump over a 6-french transjejunal plastic tube.

mon bile duct (Fig. 5B).

After meticulous dissection and debridement of the aberrant right hepatic duct stump, a $65-\mathrm{cm}$ in length transmesocolic, retrogastric Roux-en-Y limb was anastomosed termino-laterally with the aberrant biliary duct stump over a 6-French transjejunal plastic tube, implementing a fine coincidence anastomotic technique (Fig. 6). The transanastomotic tube was inserted into the jejunal lumen through the small enterotomy made for the creation of the hepaticojejunostomy and passed for about $25 \mathrm{~cm}$ distal and brought out from the bowel lumen via a small enterotomy made in the center of a double purse-string suture in the antimesenteric border of jejunal limp. The exit site of the tube was approximated in the parietal peritoneum (extraperitonalization), with a continuous circumferential stich.

Cholangiograms obtained from the transanastomotic



Fig. 7. Cholangiogram obtained from the transanastomotic tube on the fifth postoperative day depicting normal anastomosis with complete opacification of segments V, VIII and VII of the liver without signs of leakage.

tube intraoperatively and on the fifth postoperative day (Fig. 7) depicted complete opacification of segments V, VIII and VII of the liver without signs of leakage. Surgical drains removed on the 7th POD. The patient had an uneventful postoperative course and was discharged on the 12th POD. Follow-up postoperative volume-rendered MR cholangiopancreatography performed on the 20th POD depicted a normal anastomosis between the jejunal loop and the cystohepatic duct (Fig. 8). The transanastomotic tube was removed on the 60th POD. She remains well 6 months following definitive surgery.

\section{DISCUSSION}

Multiple common classified and uncommon complex 


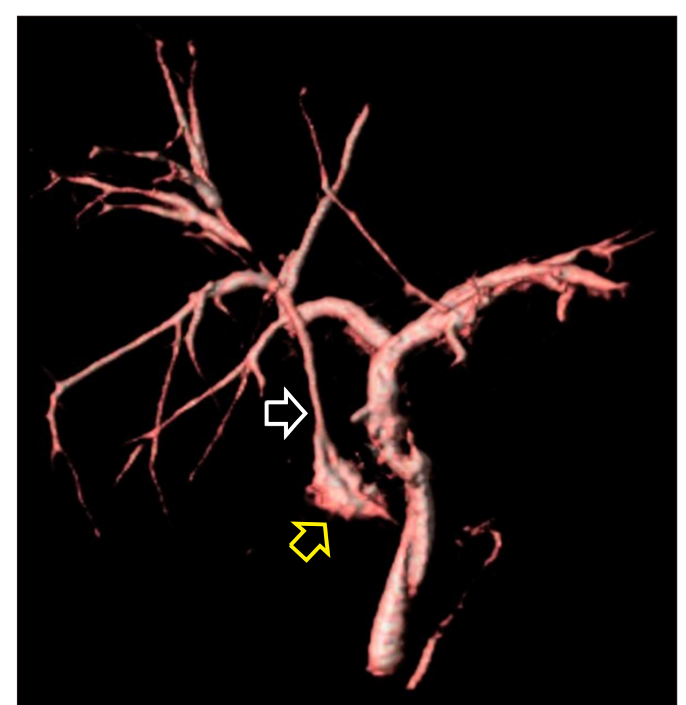

Fig. 8. Postoperative volume-rendered 3 Tesla MR cholangiopancreatography performed on the 20th POD depicting a normal anastomosis between the jejunal loop (yellow arrow) and the cystohepatic duct (white arrow).

unclassified variations of bile duct branching pattern can be encountered on various levels of the biliary tract, further intensifying the complexity of biliary interventions. In 2003, Choi et al. ${ }^{4}$ analyzing intraoperative cholangiografic data, classified the branching patterns of the biliary tract. They found that the bile duct branching patterns were atypical in $37 \%$ of cases and that the most common variation involved the RPSD, with its opening into the left hepatic duct (Choi's type 3A) being the most common. Similar findings were reported in several subsequent international studies and in a cadaveric study from our own country (Greece). ${ }^{5-7}$ In very rare instances, variant right bile duct anatomy can be complex, as in the present case, namely right inferior segmental duct opening into the left hepatic duct combined with an aberrant right hepatic duct pouring into the cystic duct-cystohepatic duct (Choi's type 7 biliary tract variation). ${ }^{4}$

Aberrant right sectoral ducts provide biliary drainage to variable segments of right hemi-liver draining directly into the common hepatic duct, common bile duct or even left hepatic duct. ${ }^{8}$ They represent a rather common anomaly of the biliary tree with an incidence ranging between $4.6 \%$ and $8.4 \%{ }^{9}$ However, opening of an aberrant right hepatic duct, formed by the junction of right anterior sectoral duct and right inferior anterior (VII) segmental duct, into the cystic duct (cysticohepatic duct) is an extremely rare bile duct branching variation that constitutes a dread- ful pitfall during open or LC. The incidence of this anatomic variation, described as "cysticohepatic duct", ranges between $1 \%$ and $2 \% .^{10,11}$ In the study by Sarawagi et al. ${ }^{3}$ aberrant segmental duct from the right lobe into the cystic duct was noted in $1.3 \%$ of 224 patients who underwent MRCP, while in the study by Ohkubo et al., who analyzed the bile duct patterns at the hepatic hilum in the surgical specimens of right-sided hepatectomy with extrahepatic bile duct resection, this variant was absent in 110 examined specimens.

When performing open or laparoscopic cholecystectomy in patients with this variation the cystic duct must be ligated between the infundibulum and the point at which the cystic duct joins the anomalous hepatic duct. Correct implementation of "critical view of safety" technique, before dividing the cystic structures can safely overcome this surgical pitfall. ${ }^{12}$

Intraoperative cholangiography (IOC) during hepatobiliary surgery, can increase the chance of recognizing bile duct variations, although its benefit in preventing BDI remains controversial. ${ }^{13}$ Use of IOC was deemed unnecessary at the original-index operation suggesting that a visual perceptual illusion leaded the surgical team to misidentification and transection of the cysticohepatic duct during dissection in Calot's triangle. At reoperation, which performed at the same institution, the bile leak has erroneously attributed to a minor biliary radical injury in the ifundibulum fossa which has unfortunately delayed diagnosis.

Isolated cysticohepatic sectoral or hepatic duct injuries are not described in the original Bismuth classification of BDI. ${ }^{14}$ However, they can be included in the Strasberg classification as either occlusion (type B) or leak (type C) from a bile duct that does not open to the common bile duct. ${ }^{15}$ Undiagnosed Strasberg's C BDI usually presents with signs of biliary peritonitis, biloma formation and biliary leak from the abdominal drains or the surgical wound ${ }^{16}$ which was the case in the present report.

Accurate diagnosis of sectoral or hepatic duct injuries is a challenge. Although endoscopic retrograde cholangiography (ERC) plays a central role in the diagnosis of BDI however, correct identification of a sectoral or hepatic BDI by ERC can achieved in less than $20 \%$ of patients. ${ }^{17,18}$ These diagnostic limitations of ERC derive from the lack of intercommunication between a transected sectoral or hepatic duct and common bile duct. In this type of injury, 
the findings of ERC are usually interpreted as normal with no recognizable biliary leaks, given that ERC fails to delineate cysticohepatic ducts when they have been clipped and/or transected as happened in the present case. However, careful evaluation of ERC can provide indirect diagnostic signs of a possible aberrant hepatic duct injury by depicting paucity of intrahepatic biliary filling in a liver segment. Notwithstanding this, endoscopy has no role in the management of Strasberg's type C BDI. ${ }^{16}$

Patients with sectoral or aberrant hepatic BDI may require additional procedures such as percutaneous cholangiography (PTC) to establish a definitive diagnosis. Percutaneous cholangiography in conjunction with external biliary drainage allows leak control, and can serve as a bridge for definitive repair. ${ }^{16}$ However, this modality is technical challenging and often impossible in cases with non-dilated biliary ducts. ${ }^{19}$ Taking this into consideration, it was decided not to attempt this approach in our patient, although some data showed that dilation of a sectoral or segmental duct is not a prerequisite for a successful PTC. ${ }^{18}$

The role of magnetic resonance cholangiopancretograpthy (MRCP) in patients with BDI is crucial. ${ }^{20}$ It provides a complete anatomical map of the biliary tract and evaluates severed ducts not communicating with the main biliary system, being an extremely useful tool in the planning of surgical reconstruction. Additionally, MRCP can confirm the presence of a variant bile duct pattern. ${ }^{21}$ In the present case, MRCP enabled not only the diagnosis of a variant anatomical bile duct pattern but also the identification of the exact site of leakage and proximal cysticohepatic duct stump providing valuable help in surgical treatment planning. Recently, contrast-enhanced MRC proved to have an increased accuracy in demonstrating bile duct anatomy and detect BDI (biliary leaks and stenosis) following hepatobiliary inerventions. ${ }^{22}$

Management of Strasberg's type C major bile duct injuries require multidisciplinary approach, preoperative accurate assessment and definitely an early surgical repair, avoiding early (peritonitis and sepsis) and late (cholangitis, secondary biliary cirrhosis, portal hypertension) life threatening complications and restoring functional disconnection of a significant healthy liver volume. Surgical management depends on timing of diagnosis, presence of intra-abdominal inflammation and con- comitant bile duct and vascular injuries. ${ }^{23}$ In the present case, before definitive surgery, concomitant bile duct and vascular injuries were ruled out and measures were taken to drain bile leakage, control local infection and inflammation and improve patient's general condition.

Major hepatic or sectoral bile duct injuries detected soon after the original surgery need a definitive repair surgery with a sectoral/hepatic bile duct jejunum Roux-en-Y anastomosis implementing a fine coincidence anastomotic technique. The use of a transanastomotic tube into the injured duct can be helpful, as happened in the present case. $^{24,25}$ To the best of our knowledge, this is the first case reporting an aberrant cysticohepatic right hepatic duct injury in the English language literature. Intraoperative BDI in the setting of unclassified, complex bile duct variations although rare, consist a devastating event which is a diagnostic and therapeutic challenge.

In summary, this report describes an extremely rare case of an isolated injury to an aberrant right hepatic duct formed by the joining of ducts from segments V, VIII and VII draining into the cystic duct (cysticohepatic duct), associated with an inferior RPSD opening to left hepatic duct during LC. The present report can serve as a useful reminder of the consistent inconsistency of biliary anatomy, alerting surgeons to beware of variant bile duct branching patterns during open or LC that constitute a dreadful pitfall for severe bile duct injuries.

\section{CONFLICT OF INTEREST}

No potential conflict of interest relevant to this article was reported.

\section{ORCID}

Konstantinos Vasiliadis: https://orcid.org/0000-0002-6964-060X

Elena Moschou: https://orcid.org/0000-0001-6620-6878

Sofia Papaioannou: https://orcid.org/0000-0003-0087-3701

Panagiotis Tzitzis: https://orcid.org/0000-0002-0362-5749

Albion Totsi: https://orcid.org/000-0003-2942-4790

Stamatia Dimou: https://orcid.org/0000-0002-8510-3409

Eleni Lazaridou: https://orcid.org/0000-0002-6602-5097

Dimitrios Kapetanos:

https://orcid.org/0000-0001-8605-9721 
Christos Papavasiliou:

https://orcid.org/0000-0002-4700-0177

\section{AUTHOR CONTRIBUTIONS}

Writing, review \& editing: KV, EL, EM, PT. Performed the operation: KV, CP, AT. Methodology-visualization: EM, SP, DK, AT, SD, KV.

\section{REFERENCES}

1. Mangieri CW, Hendren BP, Strode MA, Bandera BC, Faler BJ. Bile duct injuries (BDI) in the advanced laparoscopic cholecystectomy era. Surg Endosc 2019;33:724-730.

2. Martin RF, Rossi RL. Bile duct injuries. Spectrum, mechanisms of injury, and their prevention. Surg Clin North Am 1994;74: 781-803; discussion 805-807.

3. Sarawagi R, Sundar S, Raghuvanshi S, Gupta SK, Jayaraman G. Common and uncommon anatomical variants of intrahepatic bile ducts in magnetic resonance cholangiopancreatography and its clinical implication. Pol J Radiol 2016;81:250-255.

4. Choi JW, Kim TK, Kim KW, Kim AY, Kim PN, Ha HK, et al. Anatomic variation in intrahepatic bile ducts: an analysis of intraoperative cholangiograms in 300 consecutive donors for living donor liver transplantation. Korean J Radiol 2003;4:85-90.

5. Lyu SY, Pan KT, Chu SY, Hsu MY, Chen CM, Hung CF, et al. Common and rare variants of the biliary tree: magnetic resonance cholangiographic findings and clinical implications. J Radiol Sci 2012;37:59-67.

6. Karakas HM, Celik T, Alicioglu B. Bile duct anatomy of the Anatolian Caucasian population: Huang classification revisited. Surg Radiol Anat 2008;30:539-545.

7. Mariolis-Sapsakos T, Kalles V, Papatheodorou K, Goutas N, Papapanagiotou I, Flessas I, et al. Anatomic variations of the right hepatic duct: results and surgical implications from a cadaveric study. Anat Res Int 2012;2012:838179.

8. Ohkubo M, Nagino M, Kamiya J, Yuasa N, Oda K, Arai T, et al. Surgical anatomy of the bile ducts at the hepatic hilum as applied to living donor liver transplantation. Ann Surg 2004; 239:82-86.

9. Kullman E, Borch K, Lindström E, Svanvik J, Anderberg B. Value of routine intraoperative cholangiography in detecting aberrant bile ducts and bile duct injuries during laparoscopic cholecystectomy. Br J Surg 1996;83:171-175.

10. Turner MA, Fulcher AS. The cystic duct: normal anatomy and disease processes. Radiographics 2001;21:3-22; questionnaire 288-294.

11. Champetier J, Létoublon C, Alnaasan I, Charvin B. The cys- tohepatic ducts: surgical implications. Surg Radiol Anat 1991;13: 203-211.

12. Strasberg SM, Brunt LM. Rationale and use of the critical view of safety in laparoscopic cholecystectomy. J Am Coll Surg 2010; 211:132-138.

13. Wright KD, Wellwood JM. Bile duct injury during laparoscopic cholecystectomy without operative cholangiography. Br J Surg 1998;85:191-194.

14. Bismuth H, Majno PE. Biliary strictures: classification based on the principles of surgical treatment. World J Surg 2001;25: 1241-1244.

15. Strasberg SM, Hertl M, Soper NJ. An analysis of the problem of biliary injury during laparoscopic cholecystectomy. J Am Coll Surg 1995;180:101-125.

16. Lillemoe KD, Petrofski JA, Choti MA, Venbrux AC, Cameron JL. Isolated right segmental hepatic duct injury: a diagnostic and therapeutic challenge. J Gastrointest Surg 2000;4:168-177.

17. Kaffes AJ, Hourigan L, De Luca N, Byth K, Williams SJ, Bourke MJ. Impact of endoscopic intervention in 100 patients with suspected postcholecystectomy bile leak. Gastrointest Endosc 2005;61:269-275.

18. Perini RF, Uflacker R, Cunningham JT, Selby JB, Adams D. Isolated right segmental hepatic duct injury following laparoscopic cholecystectomy. Cardiovasc Intervent Radiol 2005;28: 185-195.

19. Perera MT, Monaco A, Silva MA, Bramhall SR, Mayer AD, Buckels JA, et al. Laparoscopic posterior sectoral bile duct injury: the emerging role of nonoperative management with improved long-term results after delayed diagnosis. Surg Endosc 2011;25:2684-2691.

20. LeBedis CA, Bates DDB, Soto JA. Iatrogenic, blunt, and penetrating trauma to the biliary tract. Abdom Radiol (NY) 2017; 42:28-45.

21. Bujanda L, Calvo MM, Cabriada JL, Orive V, Capelastegui A. MRCP in the diagnosis of iatrogenic bile duct injury. NMR Biomed 2003;16:475-478.

22. Palmucci S, Roccasalva F, Piccoli M, Fuccio Sanzà G, Foti PV, Ragozzino A, et al. Contrast-enhanced magnetic resonance cholangiography: practical tips and clinical indications for biliary disease management. Gastroenterol Res Pract 2017;2017:2403012.

23. Li J, Frilling A, Nadalin S, Radunz S, Treckmann J, Lang H, et al. Surgical management of segmental and sectoral bile duct injury after laparoscopic cholecystectomy: a challenging situation. J Gastrointest Surg 2010;14:344-351.

24. Schipper IB, Rauws EA, Gouma DJ, Obertop H. Diagnosis of right hepatic duct injury after cholecystectomy: the use of cholangiography through percutaneous drainage catheters. Gastrointest Endosc 1996;44:350-354.

25. Meyers WC, Peterseim DS, Pappas TN, Schauer PR, Eubanks $\mathrm{S}$, Murray E, et al. Low insertion of hepatic segmental duct VII-VIII is an important cause of major biliary injury or misdiagnosis. Am J Surg 1996;171:187-191. 